\title{
Surface Roughness Impact on Francis Turbine Performances and Prediction of Efficiency Step Up
}

\author{
Pierre Maruzewski ${ }^{1}$, Vlad Hasmatuchi ${ }^{1}$, Henri-Pascal Mombelli ${ }^{1}$, \\ Danny Burggraeve ${ }^{2}$, Jacob Iosfin ${ }^{2}$, Peter Finnegan ${ }^{2}$ and François Avellan ${ }^{1}$ \\ ${ }^{1}$ Ecole polytechnique fédérale de Lausanne, Laboratory of Hydraulic Machines \\ Avenue de Cour 33 bis, $\mathrm{CH}-1007$ Lausanne, Switzerland \\ ${ }^{2}$ British Columbia Hydro Generating Engineering \\ Country 6911 Southpoint Drive Burnaby, B.C. V3N 4X8 Canada
}

\begin{abstract}
In the process of turbine modernizations, the investigation of the influences of water passage roughness on radial flow machine performance is crucial and validates the efficiency step up between reduced scale model and prototype. This study presents the specific losses per component of a Francis turbine, which are estimated by CFD simulation. Simulations are performed for different water passage surface roughness heights, which represents the equivalent sand grain roughness height.

As a result, the boundary layer logarithmic velocity profile still exists for rough walls, but moves closer to the wall. Consequently, the wall friction depends not only on roughness height but also on its shape and distribution. The specific losses are determined by CFD numerical simulations for each component of the prototype, taking into account its own specific sand grain roughness height. The model efficiency step up between reduced scale model and prototype value is finally computed by the assessment of specific losses on prototype and by evaluating specific losses for a reduced scale model with smooth walls. Furthermore, surveys of rough walls of each component were performed during the geometry recovery on the prototype and comparisons are made with experimental data from the EPFL Laboratory for Hydraulic Machines reduced scale model measurements.

This study underlines that if rough walls are considered, the CFD approach estimates well the local friction loss coefficient. It is clear that by considering sand grain roughness heights in CFD simulations, its forms a significant part of the global performance estimation. The availability of the efficiency field measurements provides an unique opportunity to assess the CFD method in view of a systematic approach for turbine modernization step up evaluation. Moreover, this paper states that CFD is a very promising tool for future evaluation of turbine performance transposition from the scale model to the prototype.
\end{abstract}

Keywords: Francis Turbine, Model and Prototype Testing, CFD Simulation, Efficiency Step Up.

\section{Introduction}

In the process of turbine modernizations, the investigation of the influences of water passage roughness on radial flow machine performance is crucial and validates the efficiency step up between reduced scale model and prototype. In the past, Kurokawa studied theoretically and experimentally the roughness effects on the three dimensional boundary layer flow along an enclosed rotating disk [1]. In 1997, IEC TC4 WG18 group, convened by Tanaka, initiated its work. The WG18 IEC working group decided to revise and upgrade the scale effect formula in the existing IEC code 60995. In 2000, it incorporated the new formula for reaction turbines and pumps [2]. In 2004, Tanaka established a scale effect formula for axial flow machines [3]. At the same time, Nichtawitz emphasized that three different friction coefficients, as a function of Reynolds number and roughness, were necessary to determine the specific losses of hydraulic machines [4]. Recently, Krishnamachar and Fay synthesized analytical procedures with practical data and provided a reasonably simple computational method to obtain realistic estimation for roughness effects on the optimum efficiency of Francis turbines [5]. 
This work underlines that if rough walls are considered, the CFD approach estimates well the local friction loss coefficient with respect to the values provided by Churchill's formula [7]. It is clear that imposing sand grain roughness heights as part of the wall condition in CFD simulations makes up a significant part of the global performance estimation and prediction of efficiency step up according to the methodology depicted in Fig. 1.

The scope of the present paper is to present the methodology of prediction for the efficiency step up based on CFD numerical simulation taking into account the turbine component wall roughness and to validate this step up with respect to the available experimental results obtained from both field and model tests. First the BC Hydro Francis turbine case study and EPFL base line tests are presented. The following section describes the numerical model, the investigated simulations, as well as the corresponding validations. The numerical results of the impact of surface roughness on Francis turbine performances are presented in the results section. Before the conclusions, the prediction of efficiency step up by CFD simulation under roughness consideration is assessed.

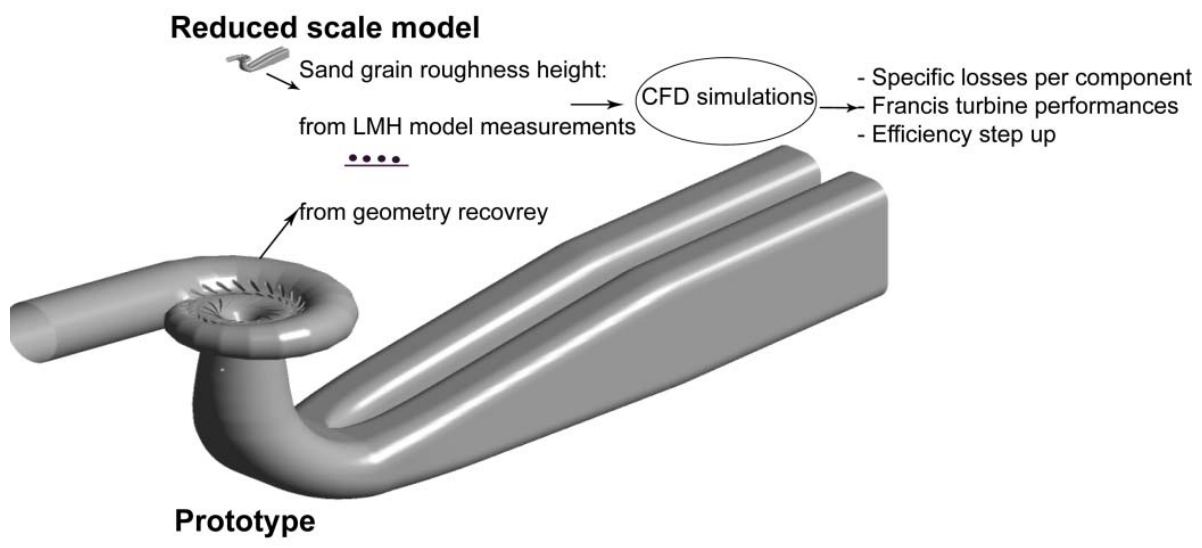

Fig. 1: Methodology for the prediction of efficiency step up

\section{Case study: the Gordon Merritt Shrum generating station}

Williston Lake, situated North-East of Vancouver, is the largest reservoir in British Colombia. The WAC Bennett Dam from British Columbia Hydro, BC Hydro, is the controlling structure for the Reservoir. The dam is $186 \mathrm{~m}$ high and 2'068 $\mathrm{m}$ long along its crest. This dam and Gordon Merritt Shrum, GMS, generating station were constructed between 1961 and 1968 . GMS houses 10 generating units that have a combined maximum output of 2'730 MW. The GMS turbines consist of medium head hydraulic Francis turbines. The case study is about turbines 1 to 5, commissioned in 1968 see Fig. 2; these turbines have a rated power of 265 MW under $161 \mathrm{mWC}$ head.

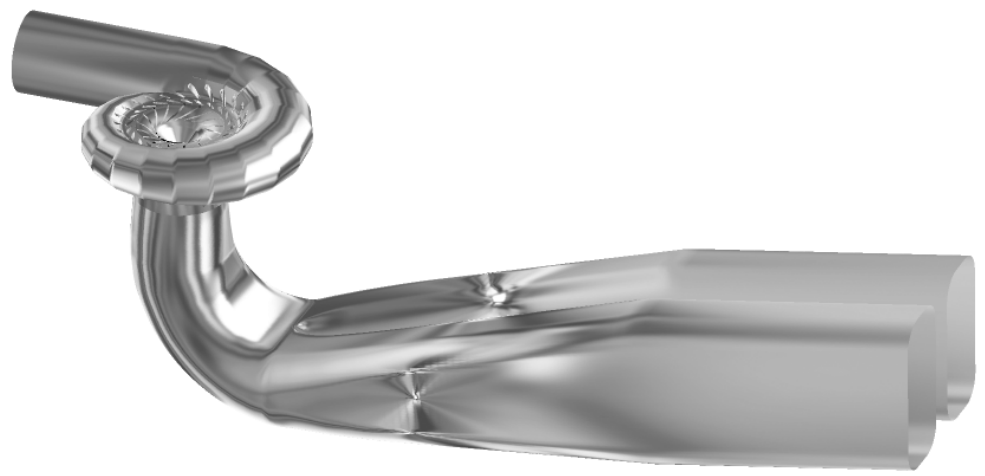

Fig. 2: Computing domain of the GMS Unit 2 Francis turbine

The EPFL was contracted by BC Hydro to carry out the geometry recovery of GMS Unit 2, in 2006, in the frame of a refurbishment project, [8]. EPFL performed a reverse engineering process which involves measuring several blades of GMS Unit 2 runner and then reconstructing it as a 3D CAD model. Each blade geometry is defined by 1'350 points using a 3D FAROC arm. Finally, a reduced scale model homologous to the prototype was manufactured and tested. Furthermore, during the geometry recovery, an evaluation of the roughness of each turbine component was performed as well. The corresponding estimated sand grain roughness heights were around hundred microns, and were not uniformly distributed. These roughness height values are used as wall roughness conditions for the CFD numerical simulation of the prototype.

GMS Unit 1 to 5 turbine model tests were performed at the EPFL PF3 test rig in 2008. The evolution of model efficiency and of prototype mechanical power is represented in Fig. 3 for the prototype net head of $161 \mathrm{~m}$. 


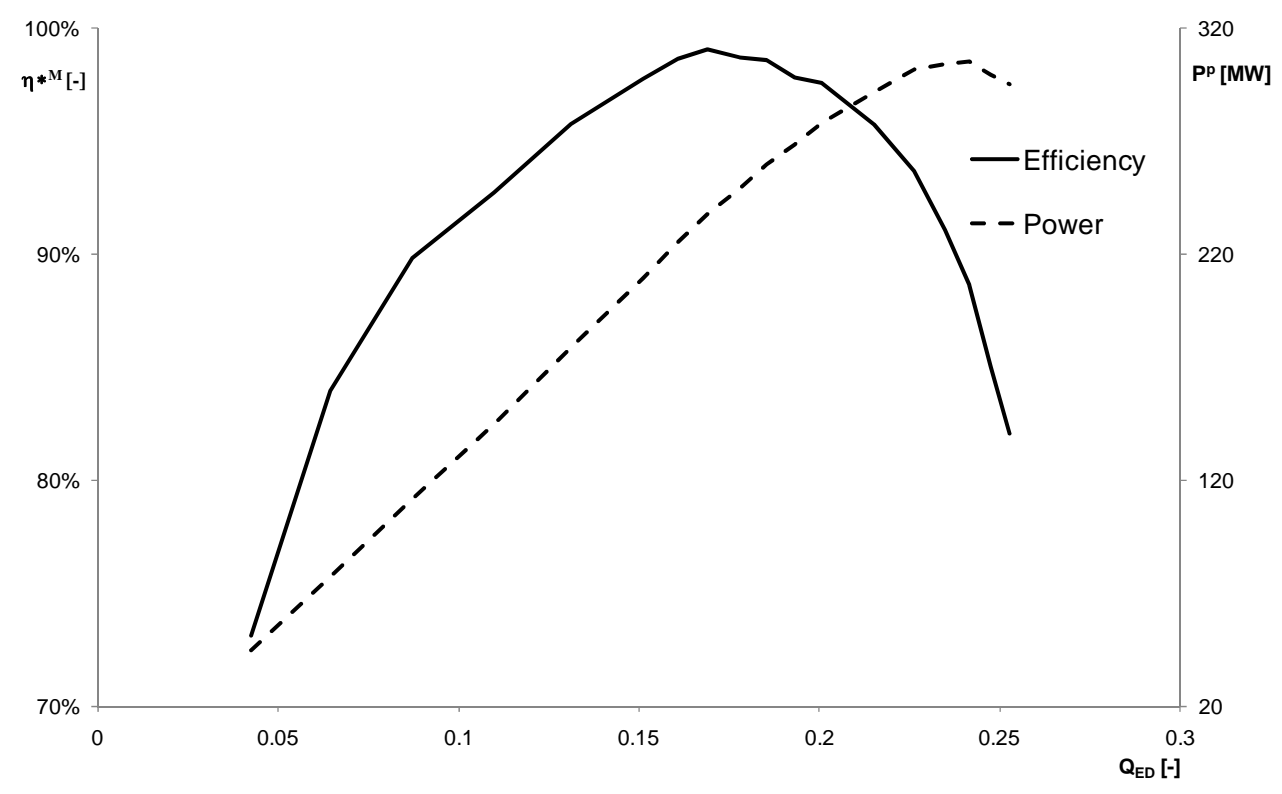

Fig. 3: Model reduced efficiency and rated power for a net head of $161 \mathrm{mWC}$ as a function the discharge factor QED [8]

\section{CFD Numerical simulations}

The Computational Fluid Dynamics numerical simulations are carried out with the ANSYS CFX-11 software, [8]. The unsteady Reynolds Averaged Navier-Stokes, URANS; equations are solved with two additional equations for modeling the turbulence. Moreover, CFX-11 features the capability for the user to impose rough walls for the treatment of local friction condition provided that the k- $\varepsilon$ turbulence model is selected. For rough walls, the boundary layer logarithmic velocity profile still exists, but moves closer to the walls. The roughness height specified is the equivalent sand grain roughness height. This is either exactly equal to the real roughness height of the surface under consideration or a function of the arithmetic mean roughness, $R a$. Therefore, the appropriate equivalent sand grain roughness height must be defined according to White [9] or Schlichting [10]. Roughness effects are accounted for by modifying the expression for $u^{+}$as follows:

$$
u^{+}=\frac{1}{\kappa} \ln \frac{y^{*}}{1+0.3 k^{+}} \quad k^{+}=y_{R} \frac{\rho}{\mu} u^{*} \quad u^{*}=C_{\mu}^{1 / 4} k^{1 / 2}
$$

With $u^{+}$representing the near wall velocity, $\kappa$ the von Karman's constant, $y^{*}$ the scalable dimensionless distance from the wall, $\mu$ the dynamic viscosity, $\rho$ the density, $C_{\mu}$, the turbulence model constant, $k$ the turbulent kinetic energy and $y_{R}$ the equivalent sand grain roughness height.

Specific energy losses and efficiency predictions are performed by the hydraulic power dissipation breakdown from the numerical simulations on the computing domain $\mathrm{V}_{\mathrm{sc}+\mathrm{v}+\mathrm{o}+\mathrm{b}+\mathrm{d}}$, represented in Fig. 4.

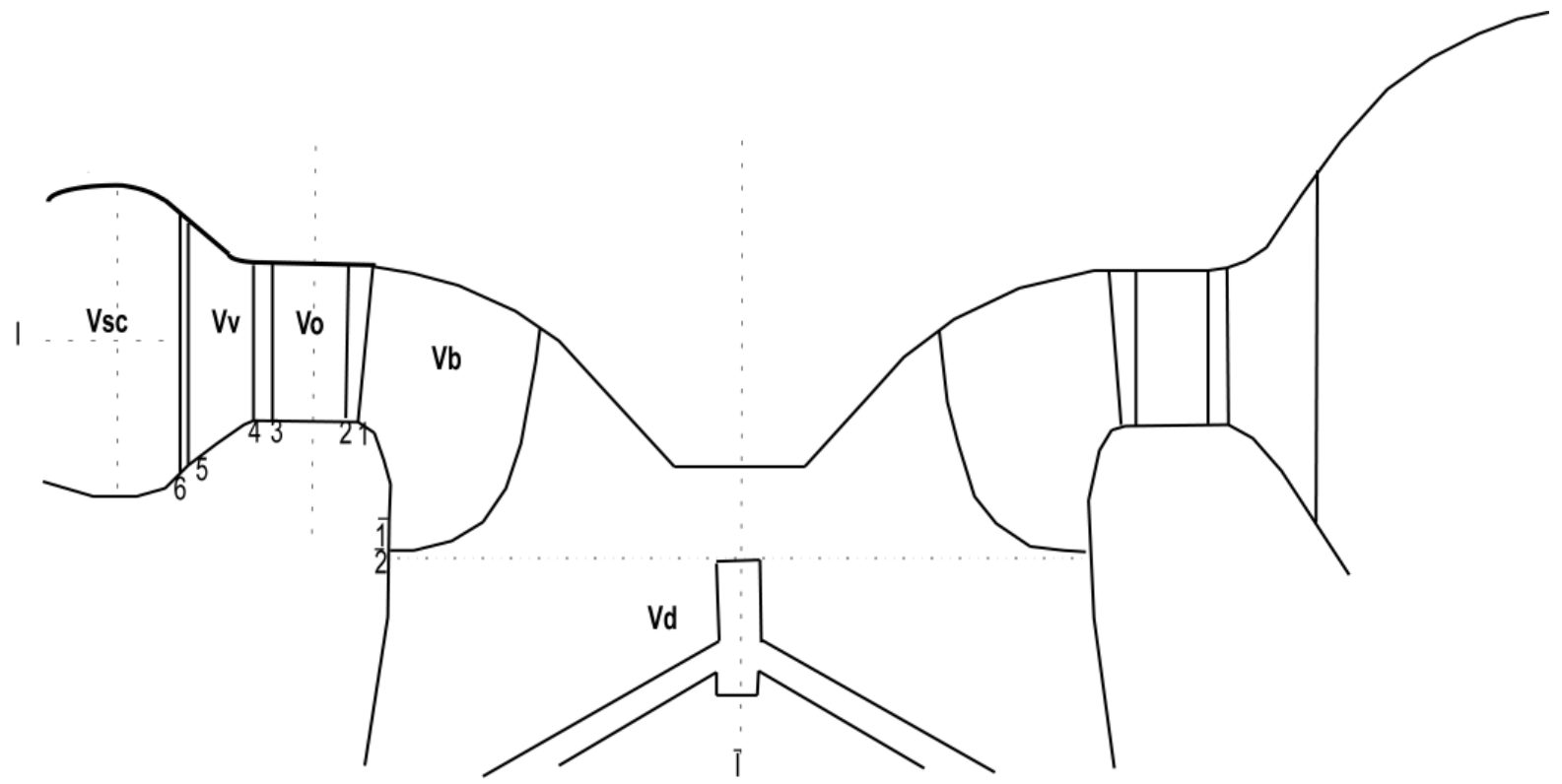

Fig. 4: Nomenclature of GMS Unit 2: -sc, the spiral casing; -v the stay vane;-o the guide vane; -b the runner; -d the draft tube 
The available hydraulic power, $P_{h}$, is the sum of the mechanical power generated by the runner, $P_{t}$ with the hydraulic power $P_{r}$ dissipated by the flow in each component of the turbine.

The following relations stands form the power flow balance in the computing domain:

$$
\begin{gathered}
P_{h}=P_{r}+P_{t}=\rho Q\left(g H_{I}-g H_{\bar{I}}\right) \\
P_{r}=P_{r s c}+P_{r v}+P_{r o}+P_{r b}+P_{r d}+P_{r q} \\
P_{t}=\omega T_{t}=\rho Q_{t} E_{t}
\end{gathered}
$$

Where $T_{t}$ is the torque exerted by the flow on the runner flow passage, $E_{t}$ the transferred specific energy, $Q_{t}$ the mass flow through the blades of the runner, and $Q$ the mass flow through a component of the hydraulic turbine.

The dissipated power, $P_{r i}$, within each $\mathrm{i}^{\text {th }}$ turbine component is defined by Equation (5)

$$
P_{r i}=\rho Q E_{r i}=\rho Q\left(g H_{I i}-g H_{\overline{I i}}\right)
$$

Where $E_{r i}$ represents the specific hydraulic energy loss in the $\mathrm{i}^{\text {th }}$ component.

The specific pressure energy, the specific potential energy and the specific kinetic energy are contributing to the specific hydraulic energy of a fluid particle as it follows

$$
\frac{p}{\rho}+g Z+\frac{C^{2}}{2}
$$

Then the average specific hydraulic energy $g H_{j}$ of a fluid in section $A_{j}$ is defined by

$$
g H_{j}=\frac{1}{Q} \int_{A_{j}}\left[\frac{p}{\rho}+g Z+\frac{C^{2}}{2}\right] \vec{C} \cdot \vec{n} d A
$$

Furthermore, the present power balance does not take into account the $P_{r q}$, the leakage power losses, and the disk friction power losses. Then, the balance of power within the runner reduced to

$$
P_{h b}=P_{t}+P_{r b}=\rho Q\left(g H_{1}-g H_{\overline{1}}\right)
$$

And from Avellan $0, P_{h b}, \mathrm{P}_{\mathrm{t}}$ and $P_{r b}$ are defined by

$$
\begin{gathered}
P_{h b}=-\int_{A_{1} \cup A_{1}} \rho(\vec{C} \cdot \vec{U}) \vec{C} \cdot \vec{n} d A-\int_{A_{1} \cup A_{1}} \rho\left[\left\{2 v \overline{\bar{D}}+\frac{\overline{\bar{\tau}}_{t}}{\rho}\right\} \cdot \vec{U}\right] \cdot \vec{n} d A+\int_{V} \rho\left\{2 v \overline{\bar{D}}: \overline{\bar{D}}+\frac{1}{\rho} \overline{\bar{\tau}} \cdot: \overline{\bar{D}}\right\} d V \\
P_{t}=-\int_{A_{1} \cup A_{1}} \rho(\vec{C} \cdot \vec{U}) \vec{C} \cdot \vec{n} d A+\int_{A_{1} \cup A_{1}}(\overline{\bar{\tau}}+\overline{\bar{\tau}}) \vec{n} \cdot \vec{U} d A \\
P_{r b}=\int_{V} \rho\left\{2 v \overline{\bar{D}}: \overline{\bar{D}}+\frac{1}{\rho} \overline{\bar{\tau}}: \overline{\bar{D}}\right\} d V
\end{gathered}
$$

The hydraulic power balance in the computing domain could be defined by the sum of specific energy losses as

$$
\rho Q E=\rho Q\left(E_{r s c}+E_{r v}+E_{r o}+E_{t}+E_{r b}+E_{r d}\right) \quad \text { with } E=E_{t}+\sum E_{r x}
$$

The specific hydraulic energy efficiency, $\eta_{e}$, of the hydraulic turbine is defined by

$$
\eta_{e}=\frac{E_{t}}{E_{t}+\Sigma E_{r x}}=1-\frac{\Sigma E_{r x}}{E_{t}+\sum E_{r x}}
$$

The computing domain includes all the machine components, i.e. the spiral casing, the stay vane, the guide vane, the runner and the draft tube with a tripod in the draft tube cone. The fluid-fluid interface with stage option is imposed at the interface between the guide vane and the runner domains and between the runner and the draft tube cone domains as well. In the simulations, walls are considered as rough and then the specific sand grain roughness height per component is taken into account. The characteristics of the mesh for the computing domain $\mathrm{V}_{\mathrm{sc}+\mathrm{v}+0+\mathrm{b}+\mathrm{d}}$ are summarized in Table 1 for both cases, the prototype, p, and the reduced scale model, $\mathrm{m}$. 
Table 1: Mesh characteristics of the computing domain $\mathrm{Vsc}+\mathrm{v}+\mathrm{o}+\mathrm{b}+\mathrm{d}$

\begin{tabular}{|c|c|c|c|c|c|}
\hline Component & $\begin{array}{c}\text { Number of } \\
\text { Nodes }\end{array}$ & $\begin{array}{c}\text { Sand } \\
\text { Roughness Height }\end{array}$ & Inlet & " Outlet & Topology \\
\hline Spiral casing & 640’071 & $\begin{array}{l}\text { p: } 1.510^{-4} \mathrm{~m} \\
\mathrm{~m}: 7.210^{-7} \mathrm{~m}\end{array}$ & \multirow{6}{*}{$\begin{array}{c}\text { Net head } \\
\text { p: 161mWC } \\
\text { m: } 30 \mathrm{mWC}\end{array}$} & \multirow{6}{*}{$\begin{array}{c}\text { Relative } \\
\text { pressure } \\
\text { equal to } \\
0 \mathrm{~Pa}\end{array}$} & \multirow{6}{*}{ Structured } \\
\hline Stay vane & $810 ’ 450$ & $\begin{array}{l}\text { p: } 1.010^{-4} \mathrm{~m} \\
\mathrm{~m}: 1.610^{-7} \mathrm{~m}\end{array}$ & & & \\
\hline Guide vane & 1’073’088 & $\begin{array}{l}\text { p: } 1.2510^{-5} \mathrm{~m} \\
\mathrm{~m}: 2.810^{-7} \mathrm{~m}\end{array}$ & & & \\
\hline Runner & 1’757'273 & $\begin{array}{l}\text { p: } 2.510^{-5} \mathrm{~m} \\
\mathrm{~m}: 5.810^{-7} \mathrm{~m}\end{array}$ & & & \\
\hline Draft tube & 928’268 & $\begin{array}{l}\text { p: } 5.010^{-5} \mathrm{~m} \\
\mathrm{~m}: 7.010^{-7} \mathrm{~m}\end{array}$ & & & \\
\hline $\mathrm{Vsc}+\mathrm{v}+\mathrm{O}+\mathrm{b}+\mathrm{d}$ & 5’209'150 & - & & & \\
\hline
\end{tabular}

The Reynolds number, Re, increases from $5.710^{6}$ for reduced scale model to $1.810^{8}$ for prototype.

Two cases of wall condition for numerical simulations on the prototype are investigated. In one case, the sand grain roughness height is considered as a linear function of the arithmetical mean roughness, $R_{a}$, by the following relation:

$$
y_{R}=k \cdot R_{a} \text {, where } k=0.1,1 . .6 \quad y_{R} \in[2.5: 150] \mu m
$$

In the other case, real sand grain roughness heights, taken from the geometry recovery and described in

Table 1, are applied to each prototype component. Moreover, two severe roughness heights conditions are also applied.

The quality of grids is estimated by evaluating the sensitivity of the pressure coefficient $C_{p}$ versus three kinds of mesh defined from the coarsest to the finest. The properties and the error of these meshes applied to the draft tube are presented in Table 2 . Then, all the following results are performed on the medium mesh with an error of $\pm 0.8 \%$ for predicting $C_{p}$ value.

Table 2 Mesh characteristics of coarse, medium and fine grids for the draft tube

\begin{tabular}{c|c|c|c}
\hline \hline$C_{p}$ Definition & Mesh & Number of Nodes & Sensitivity of $C_{p}$ \\
\hline \multirow{2}{*}{$C_{p}=2 \frac{p-p_{\overline{1} e}}{\rho C_{m \overline{1} e}^{2}} \quad C_{m \overline{1} e}=\frac{4 Q}{\pi D_{\overline{1} e}^{2}}$} & coarse & $395 ' 208$ & $3.4 \%$ \\
\cline { 3 - 4 } \cline { 3 - 4 } & medium & $928 ' 268$ & $0.8 \%$ \\
\hline
\end{tabular}

\section{Influence of Roughness on Francis Turbine Efficiency and Step up Prediction}

Regarding the ratio of specific losses over specific hydraulic energy, CFD numerical simulations allow for the definition of the limit between "admissible roughness" and "hydraulically smooth" wall as described by Nichtawitz and Tanaka in 2004[4]. They have first defined the lower limit of roughness height and called it "Admissible Roughness". All the walls with a roughness height under this limit is considered as "Hydraulically Smooth", as shown in Fig. 5.

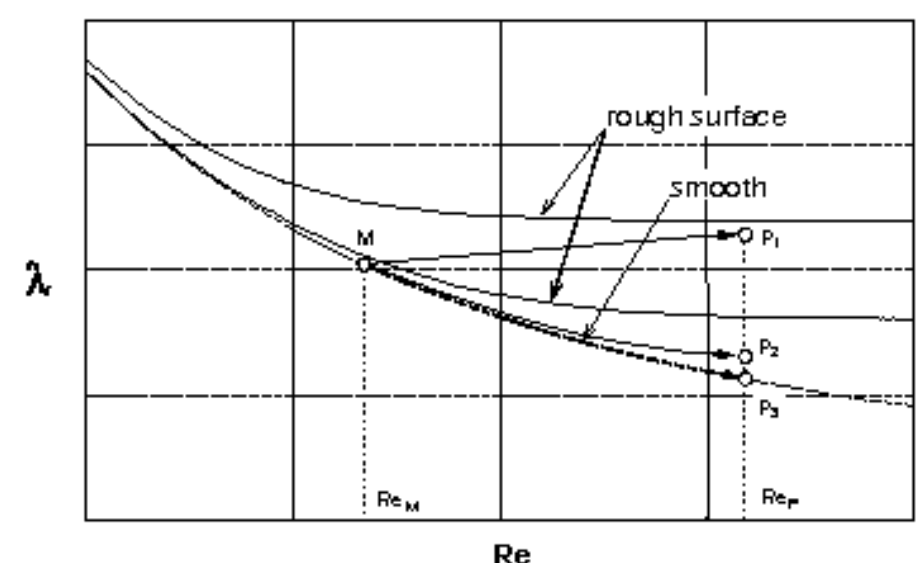




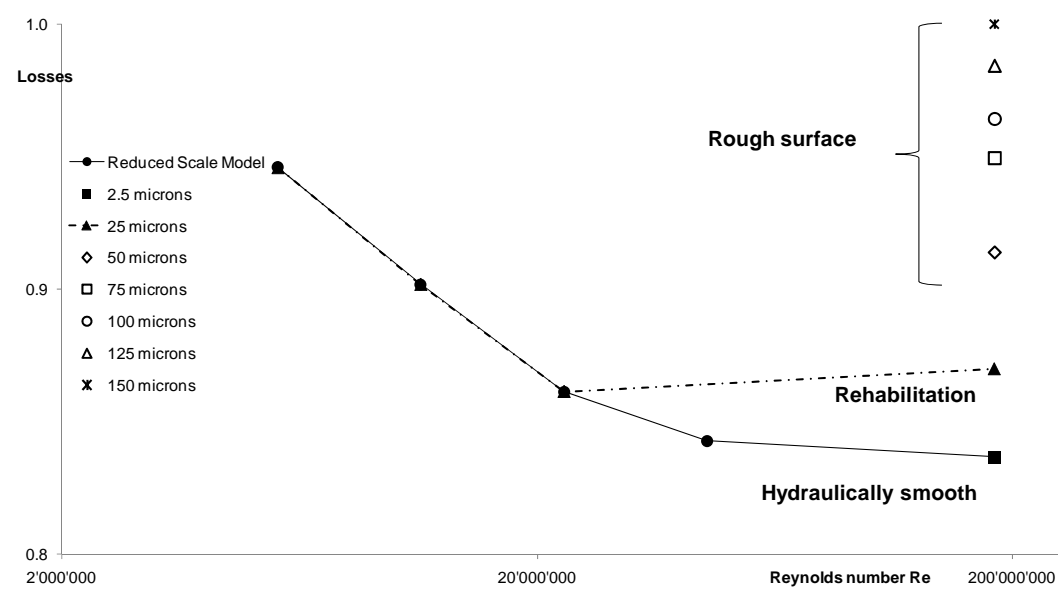

Fig. 5: Definition of hydraulically smooth surface and rough surface: on the top from Nichtawitz's analysis 0 and on the bottom from EPFL CFD simulations

The influence the wall roughness height on the specific losses prediction is then investigated by increasing the sand grain roughness height from 2.5 microns to 150 microns. According to the Fig. 5, the wall of the prototype can be considered as hydraulically smooth for sand grain roughness heights smaller than 25 microns, and as rough for sand grain roughness heights greater than 25 microns. Furthermore, the power plant operator considers that after sanding and painting an existing rough prototype, the resulting sand grain roughness height becomes equivalent to a $R_{a}$ of 10 microns; this value being characteristic of the rehabilitated machine roughness.

For all solid walls of the prototype, the impact of surface roughness on turbine performance is carried out by linearly increasing the sand grain roughness heights from 2.5 microns to 150 microns, see Fig. 6 . First, over prediction of efficiency values at the best operating point and for low discharge are observed if hydraulically smooth surfaces are considered. These over predictions have already been noticed when simulations with smooth walls are performed [13] with respect to the values provided by the Churchill's empirical loss formula [7]. Then, numerical results in the Fig. 6 show the evolution of the specific hydraulic energy efficiency versus the sand grain roughness height and versus the discharge. It is noticed that this efficiency does not quickly decrease as the roughness increases.

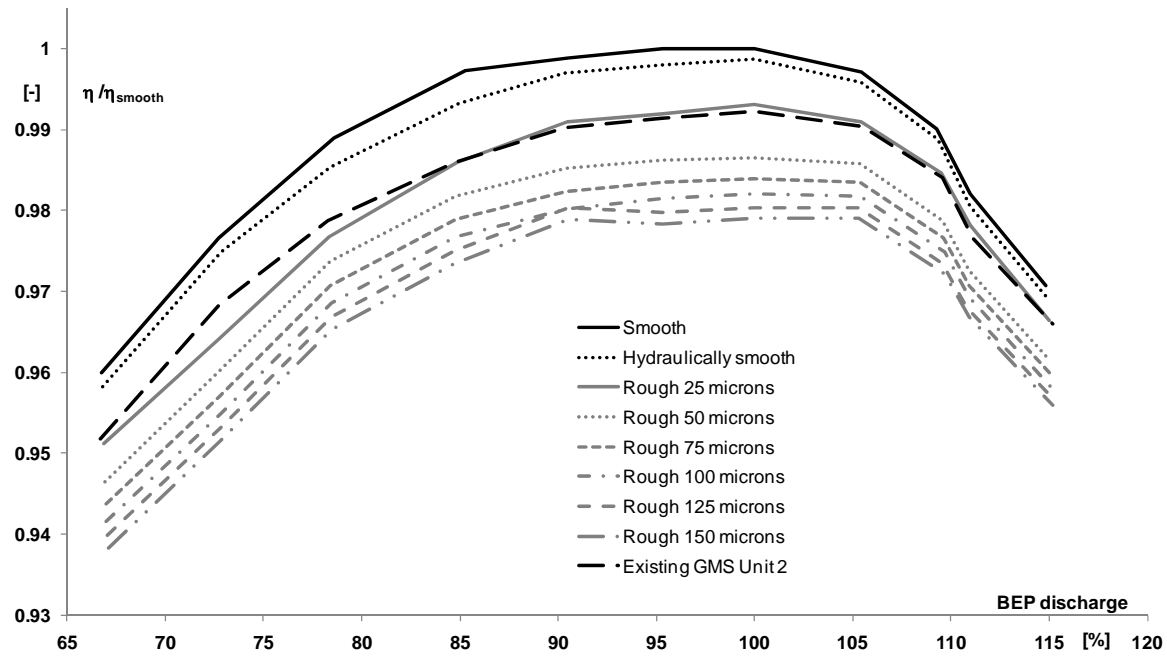

Fig. 6: Surface roughness impact on the GMS turbine specific energy efficiency

In the second case of simulations with the real sand grain roughness height per component of real GMS Unit 2 prototype, the major difficulty consists of finding the correct CFD value for the roughness height. In fact, on the real prototype, roughness is not uniformly distributed on the component walls. Some pieces of walls are smooth while others are not. However, for the CFD numerical simulations, an uniform sand roughness height should be specified to the solver. According to this constraint, it is decided to impose an averaged value of the real sand grain roughness height per component as shown in Table 1 . Hence, in the real case of GMS Unit 2, the specific energy efficiency with real sand roughness height per component is equivalent to a prototype with an uniform sand grain roughness height of 25 microns per component.

Moreover, the impact of surface roughness on specific losses per component is presented in Table 3 and in Fig. 7 for the best efficiency operating point. Three cases are investigated:

- Ideal smooth walls;

- $\quad$ Slightly rough walls with an uniform sand grain roughness height of 10 microns; 
- And finally a value corresponding to the medium measured GMS walls roughness.

The second case corresponds to a prototype condition after rehabilitation by sandblasting and painting. Performing CFD simulations with ideal smooth walls over predicts results around $1 \%$. However, rehabilitating a medium rough machine allows a decrease of $0.93 \%$ of total specific losses. Finally, the analysis of the results made apparent that it is more beneficial to rehabilitate the guide vane and the runner, than the spiral casing, the stay vanes or the draft tube.

Table 3: Surface roughness impact on specific losses per component

\begin{tabular}{|c|c|c|c|c|c|c|}
\hline $\begin{array}{l}\text { Prototype wall } \\
\text { roughness status }\end{array}$ & $\begin{array}{c}\text { Spiral } \\
\text { Casing } \\
{[\%]}\end{array}$ & $\begin{array}{l}\text { Stay } \\
\text { Vane } \\
{[\%]}\end{array}$ & $\begin{array}{c}\text { Guide } \\
\text { Vane } \\
{[\%]}\end{array}$ & $\begin{array}{c}\text { Runner } \\
{[\%]}\end{array}$ & $\begin{array}{c}\text { Draft } \\
\text { Tube } \\
{[\%]}\end{array}$ & $\begin{array}{c}\text { Total } \\
\text { Losses } \\
{[\%]}\end{array}$ \\
\hline Smooth & 0.17 & 1.05 & 1.73 & 1.35 & 0.69 & 4.99 \\
\hline Rehabilitation $10 \mu \mathrm{m}$ & 0.25 & 1.14 & 2.01 & 1.86 & 0.81 & 6.07 \\
\hline Medium rough GMS & 0.28 & 1.23 & 2.34 & 2.29 & 0.86 & 7.0 \\
\hline $\begin{array}{r}\text { Saving After } \\
\text { Rehabilitation }\end{array}$ & 0.03 & 0.09 & 0.33 & 0.43 & 0.05 & 0.93 \\
\hline
\end{tabular}
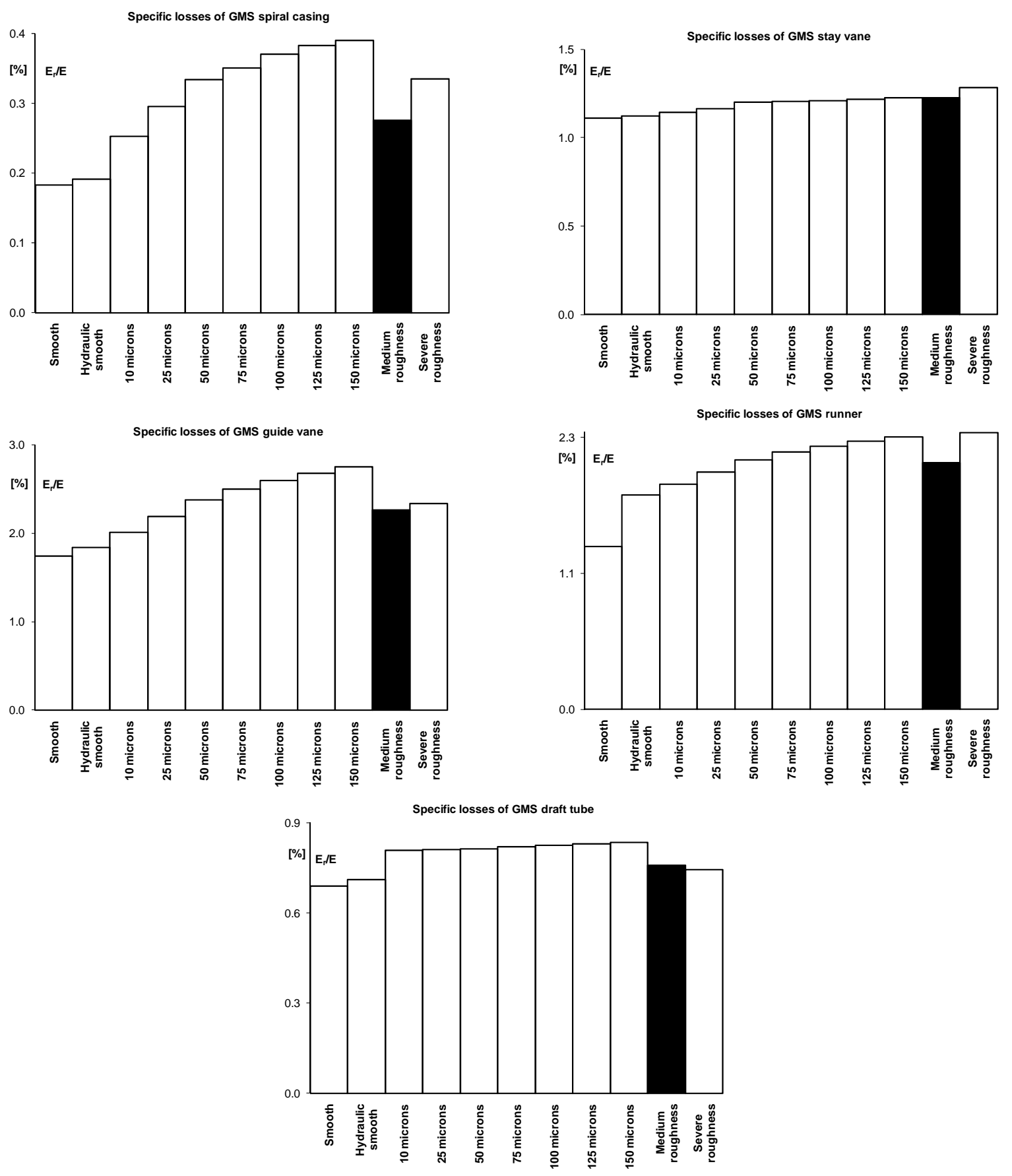

Fig. 7: Specific losses per component per sand grain roughness height 
The efficiency step up between reduced scale model and prototype is finally predicted by the analysis of the specific hydraulic energy losses on the prototype and on a reduced scale model. Then, comparisons are made with the experimental data of the reduced model base line tests as presented in Fig. 8. The CFD numerical simulations over predict the efficiency for low discharge and under predict it for high discharge. In fact, considering the breakdown of specific energy losses per component and comparing the resulting efficiency with the efficiency scale established by Osterwalder,[14], it is clear that disk friction losses and volumetric losses take a significant part for low discharge. If the CFD numerical prototype efficiency is corrected by taking into account these losses, estimated to $2 \%$ the CFD numerical and experimental efficiency curves are in a good agreement, see Fig. 9.

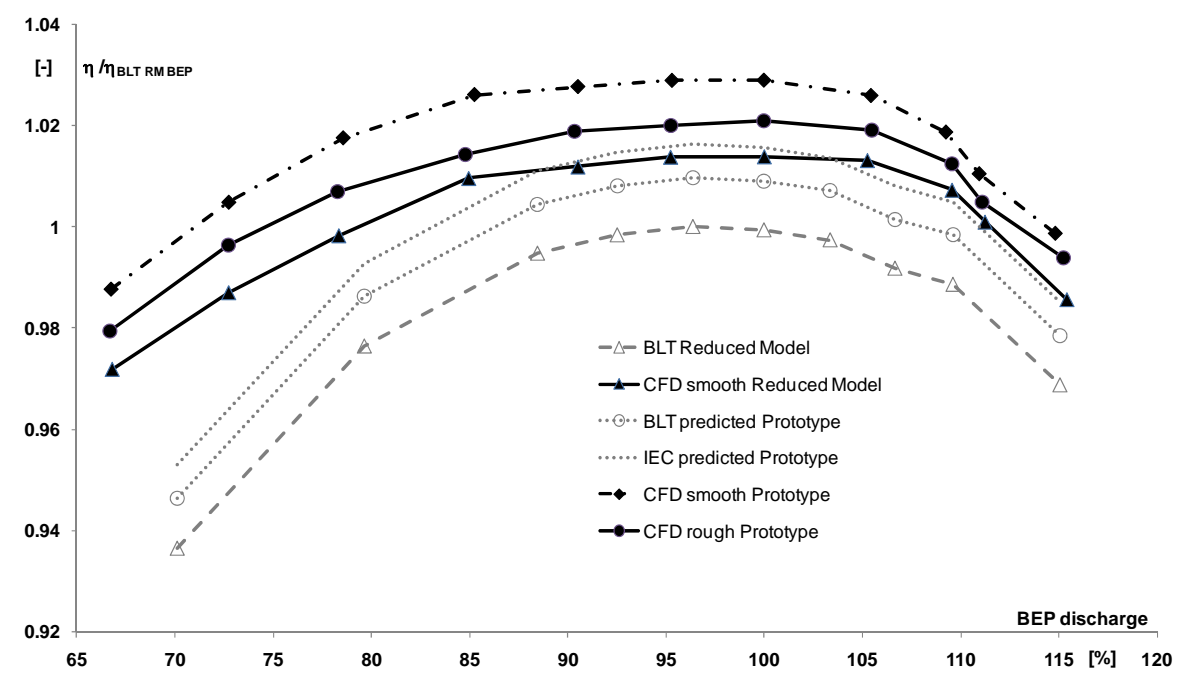

Fig. 8: Prediction of efficiency as a function of the flow rate

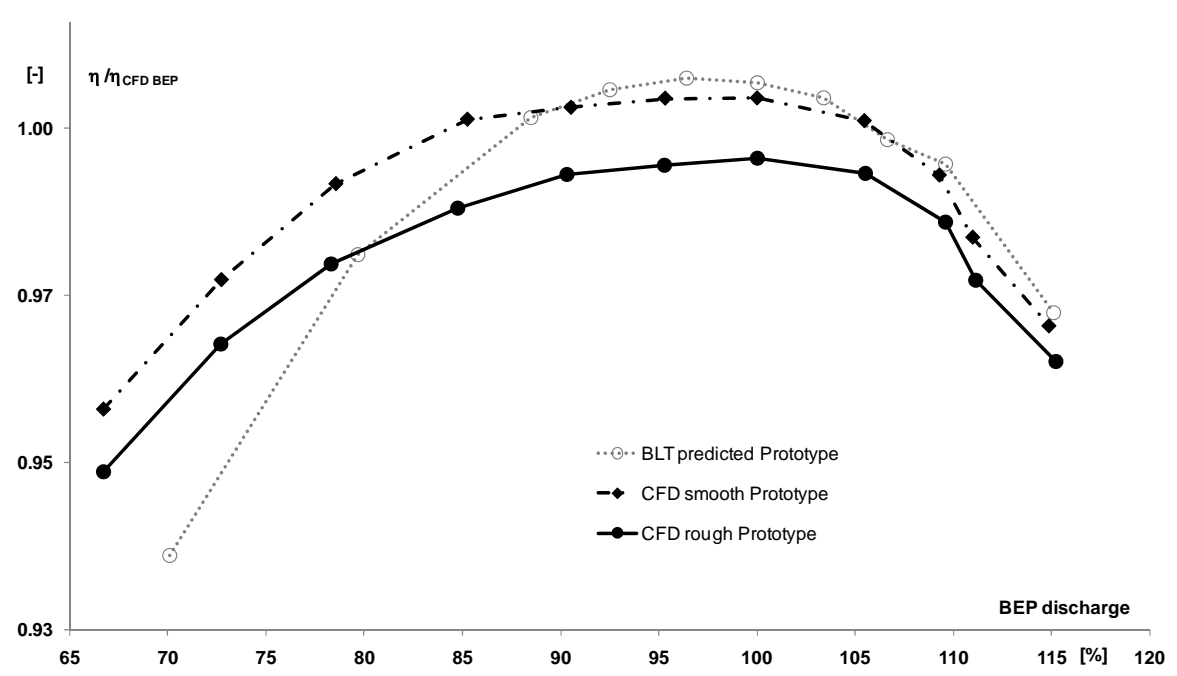

Fig. 9: Corrected energy efficiency by taking account disk and gap losses

Furthermore, the experimental efficiency of the reduced scale model allows predicting the uniform efficiency step up about 0.89\%. But, for CFD results under real roughness conditions, the numerical efficiency step up reaches $0.64 \%$ at the best efficiency operating point, see Table 4.

Table 4: Wall roughness impact on efficiency step up

\begin{tabular}{cc}
\hline \hline Cases & Efficiency Step Up \\
\hline Base line tests to rough GMS - IEC code & $+0.89 \%$ \\
CFD reduced model to rough GMS & $+0.64 \%$ \\
CFD reduced model to rehabilitation 10 $\mu \mathrm{m}$ & $+0.81 \%$ \\
CFD reduced model to smooth GMS & $+1.37 \%$ \\
\hline
\end{tabular}


Firstly, it is observed that the efficiency step up takes positive and negative values. A positive efficiency step up means that the impact of surface roughness remains weak to keep acceptable performances. Whereas, a negative efficiency step up shows that the impact of roughness is so high that the performances considerably decrease.

Moreover, considering an ideal smooth wall prototype with $y_{R}$ close to 0 microns, the efficiency step up is $+1.37 \%$. For the rehabilitation case with $y_{R}$ around 10 microns, the value of efficiency step up is around $+0.81 \%$. Finally, for a severe roughness case, the efficiency step up negatively decreases to $-0.15 \%$, see Fig. 11 . In this figure, it is shown how the GMS Unit 2 can be evolved from existing situation to rehabilitation or to stronger roughness impact.

The analysis of the impact of roughness height increases on prediction of the efficiency step up is then performed for the best efficiency operating point; see Fig. 10 and Fig. 11. An efficiency step up scale versus uniform sand grain roughness height allows for understanding of the impact of wall roughness on the efficiency step up, Fig. 10. The horizontal axis describes the uniform sand grain roughness height $y_{R}$ from 0 micron, representing a smooth wall to 150 microns, meaning a severe rough wall. The real rough GMS prototype efficiency step up is compared to the rehabilitated cases, 10 and 25 microns sand grain roughness heights, and to very severe rough walls, from 0.5 to $1 \mathrm{~mm}$ sand grain roughness heights, Fig. 11.

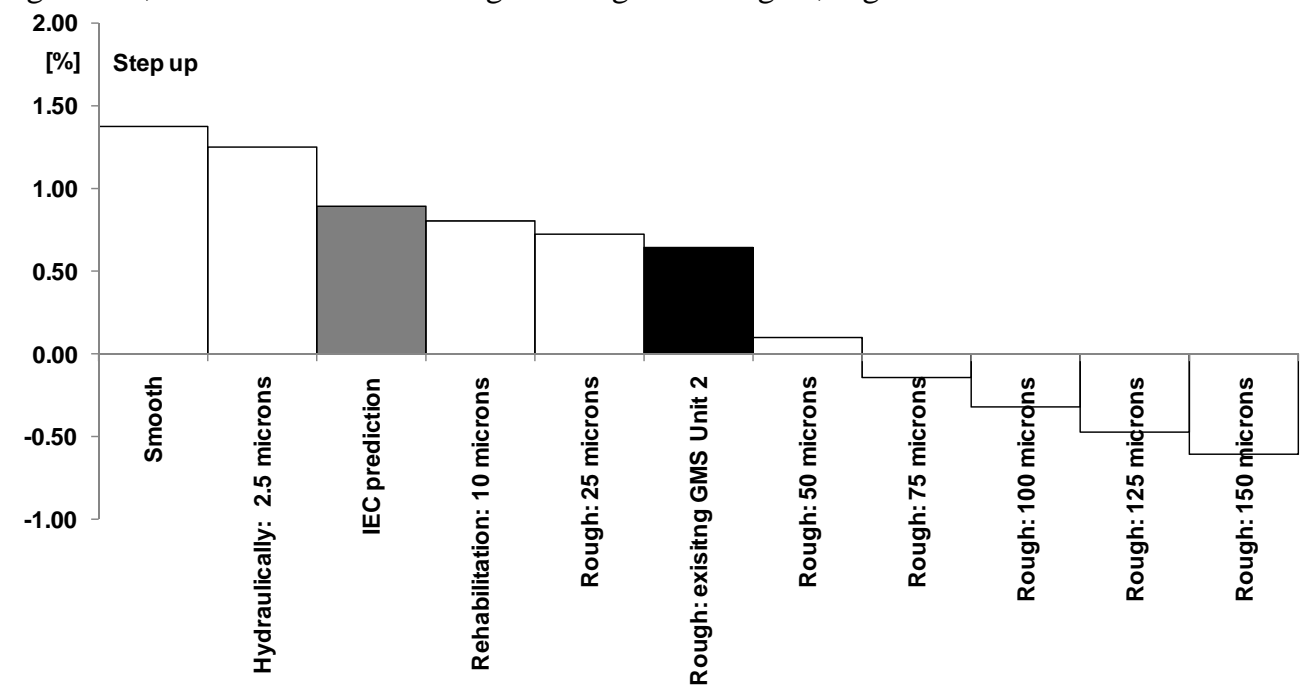

Fig. 10: Efficiency step up versus sand grain roughness height

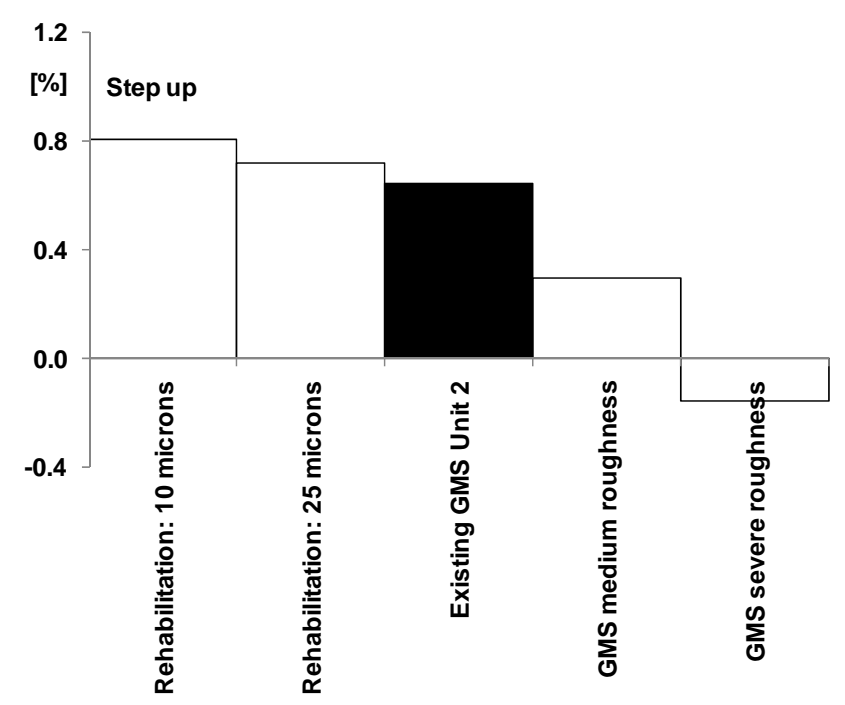

Fig. 11: Efficiency step up versus sand grain roughness height for GMS Unit 2

\section{Conclusion}

This study underlines that if rough walls are considered, the CFD approach is well predicting the total and local specific hydraulic energy losses. It is clear that modeling sand grain roughness heights in CFD predictions makes a significant impact on the global performance estimation. By this way, it is stated that CFD is a very promising tool for future evaluation of turbine performances. Moreover, the availability of the efficiency field measurements provides an unique opportunity to assess the CFD method in view of a systematic approach for turbine modernization step up evaluation.

Next features of the prediction of efficiency step up by CFD simulations are:

- $\quad$ Taking account the leakage loss;

- $\quad$ Taking account the auxiliary water passages as friction disk and labyrinth;

- Using real sand grain roughness heights of exsiting machines from manufacturers' databases. 


\section{Acknowledgments}

The authors would like to express sincere gratitude to the staff of the EPFL Laboratory for Hydraulic Machines for its support and advices to well lead this work.

\section{Nomenclature}

\begin{tabular}{|c|c|c|c|}
\hline$A_{j}$ & Cross-section $\left[\mathrm{m}^{2}\right]$ & $P_{r q}$ & Leakage power loss [MW] \\
\hline$\vec{C}$ & Fluid Velocity $\left[\mathrm{ms}^{-1}\right]$ & $p$ & Fluid Pressure [Pa] \\
\hline$C_{\mu}$ & Turbulence model constant $=0.09$ & $Q_{x}$ & Mass flow per component $x\left[\mathrm{~m}^{3} \mathrm{~s}^{-1}\right]$ \\
\hline$\overline{\bar{D}}+\overline{\bar{\tau}}_{t}$ & Stress and turbulent tensors & $T_{t}$ & Torque $[\mathrm{Nm}]$ \\
\hline$D_{\overline{1} e}$ & Component Diameter [m] & $u^{+}$ & Near wall velocity $\left[\mathrm{ms}^{-1}\right]$ \\
\hline$E_{t}$ & Transferred specific energy $\left[\mathrm{m}^{2} \mathrm{~s}^{-2}\right]$ & $y_{R}$ & Equivalent sand grain roughness height $[\mu \mathrm{m}]$ \\
\hline$E_{r x}$ & Specific hydraulic energy loss per component $x\left[\mathrm{~m}^{2} \mathrm{~s}^{-2}\right]$ & $y^{*}$ & Scalable dimensionless distance from the wall [-] \\
\hline$g H_{j}$ & Specific hydraulic energy in a section $A_{j}\left[\mathrm{~m}^{2} \mathrm{~s}^{-2}\right]$ & $\vec{U}$ & Rotational Velocity $\left[\mathrm{ms}^{-1}\right]$ \\
\hline$k$ & Turbulent kinetic energy $\left[\mathrm{m}^{2} \mathrm{~s}^{-2}\right]$ & $\eta_{e}$ & Specific hydraulic energy efficiency [-] \\
\hline$P_{h}$ & Hydraulic power [MW] & $\kappa$ & Von Karman's constant $=0.41$ \\
\hline$P_{t}$ & Mechanical power generated by the runner [MW] & $\rho$ & Fluid Density $\left[\mathrm{kg} \mathrm{m}^{-3}\right]$ \\
\hline & Hydraulic power dissipated by the flow [MW] & & \\
\hline
\end{tabular}

\section{References}

[1] Kurokawa J., Toyokura T., Shinjo M., Matsuo K., 1978, "Roughness effects on the flow along an enclosed rotating disk," bulletin of JSME, Vol. 21, No. 162, pp. 1725-1732.

[2] Tanaka H., Nichtawitz A., 2000. “New scale effect formula being studied for future IEC code,” IAHR Symposium, Charlotte.

[3] Tanaka H., Nichtawitz A., June 29-July 2, 2004, "Scale effect formula for future IEC code its theoretical background and features," IAHR Symposium, Stockholm.

[4] Nichtawitz A., Tanaka H., June 29-July 2, 2004, "Derivation of formulae for future IEC code on scale effects,” IAHR Symposium, Stockholm.

[5] Krishnamachar P., Fay A., 2007, "The effect of surface roughness, International Water Power and Dam Construction, www.waterpowermagazine.com.

[6] Maruzewski P., Hasmatuchi V., Mombelli H.-P., Burggraeve D., Iosfin J., Finnegan P. and Avellan F., 2008, "Surface roughness impact on Francis turbine performances and prediction of efficiency step up," 24th IAHR Symposium on Hydraulic Machinery and Systems, Foz do Iguassu, Brasil.

[7] Churchill S.W., 1988, Viscous Flows, “The practical use of theory,” Butterworth Ser Chem Engng, ISBN 0-409-95185-4.

[8] EPFL, 2006, British Columbia Hydro, “Geometry recover of Francis turbine,” technical report, Lausanne, Switzerland.

[9] Lechner R., Menter F. R., 2008, “Treatment of rough wall on CFX-11, ANSYS technical report,” Germany.

[10] White M., 1979, “Viscous Fluid flow,” Mac Graw-Hill.

[11] Schlichting H., 1979, "Boundary layer,” Mac Graw Hill.

[12] Avellan F., 2005, Cours de turbomachines hydrauliques, équations des turbomachines. Cours, EPFL.

[13] Maruzewski P., Avellan F., 2008, “Roughness analysis,” EPFL technical report, Lausanne, Switzerland.

[14] Osterwalder J., Hippe L., 1984, "Guidelines for efficiency scaling process of hydraulic turbomachines with different technical roughnesses of low passages,” Journal Hydraulic Research, Vol. 22, No. 2, pp. 77-10. 